\title{
Nahrung und Nahrungserwerb des Ziesels
}

\author{
Barbara HERZIG-STRASCHIL
}

\begin{abstract}
Herzig-Straschil B., 1976: Nahrung und Nahrungserwerb des Ziesels. Acta theriol., 21, 7: 131-139. [Mit 2 Tabellen u. 1 Abb.].

The feeding habits of the ground souslik Citellus citellus ( $\mathrm{L}$ in$\mathrm{n}$ a e us, 1766) are discussed. Lists of plants eaten, were based entirely on field observations; determinations of animals (remains) ingested were made in the following ways: field observations and collections of food remains; identification of chitinous remains in faeces and in stomach contents. It appears evident that the sousliks show a preference for and actively seek particular types of food, although they are capable of utilizing a wide variety of both plant and animal nourishment. Their main diet are different parts of plants. Among their animal diet Coleoptera are dominant followed by Lepidoptera (caterpillars) and Saltatoria. All food particles are taken with the mouth first - but the forepaws help handling them.

[c/o Säugetiersammlung, Naturhistorisches Museum, A-1014 Wien, Postfach 417, Burgring 7. Osterreich].
\end{abstract}

\section{EINLEITUNG}

Die Nahrung von Citellus citellus (L in na e us, 1776) wurde bisher mit unterschiedlicher Genauigkeit behandelt, wobei die Ergebnisse aus Beobachtungen im Freiland oder in Gefangenschaft, bzw. aus Untersuchungen von Magen- und Backentascheninhalten resultieren; auch durch Ziesel verursachte Schäden in der Landwirtschaft werden besprochen (B a u e r, 1960; Brinkmann, 1951; Calinescu, 1934; Grulich, 1960; M ohr, 1929; P a kizh, 1958; P es hev, 1955, R užić-Petrov, 1950; T u r č ek, 1955, 1963 und 1964, W e r th, 1934).

Die folgenden Ergebnisse der Untersuchungen über Nahrung und Nahrungserwerb des Ziesels in Österreich stammen aus einem bisher wenig behandelten Teil des Verbreitungsgebietes und stellen eine Erweiterung des bisher bekannten Materials dar.

Kolonien von C. citellus findet man im Osten Österreichs (Abb. 1) vor allem auf bewachsenen Feldwegen und Rainen, trockenen Wiesen und Weiden, und vereinzelt auch in Klee- und Luzernefeldern, sowie in Weingärten. Die Populationsdichte und Arealgröße sind in den einzelnen Biotopen allerdings sehr unterschiedlich. Die Lochdichte, die nur sehr beschränkt ein $\mathrm{Maß}$ für die Individuendichte darstellt, reicht von 500-700 Löchern/ha auf niedrig bewachsenen Feldwegen (hier aber nur in kleiner. Gruppen verteilt), über ausgedehnte Vorkommen auf Wiesen und 
Weiden mit 250 - 500 Löchern/ha, bis zu sehr kleinräumigen Vorkommen in Weingärten mit 175 und weniger Löchern/ha. Hauptmerkmal all dieser Biotope ist neben weitgehender Trockenheit fehlende oder seltene (Kleeund Luzernefelder, Weingärten) Bodenbearbeitung, sowie eine überwiegende Vegetationshöhe von maximal $10-20 \mathrm{~cm}$; die durchschnittliche Bodenbedeckung beträgt mindestens etwa 70\%. Extreme Ausnahmen bil-

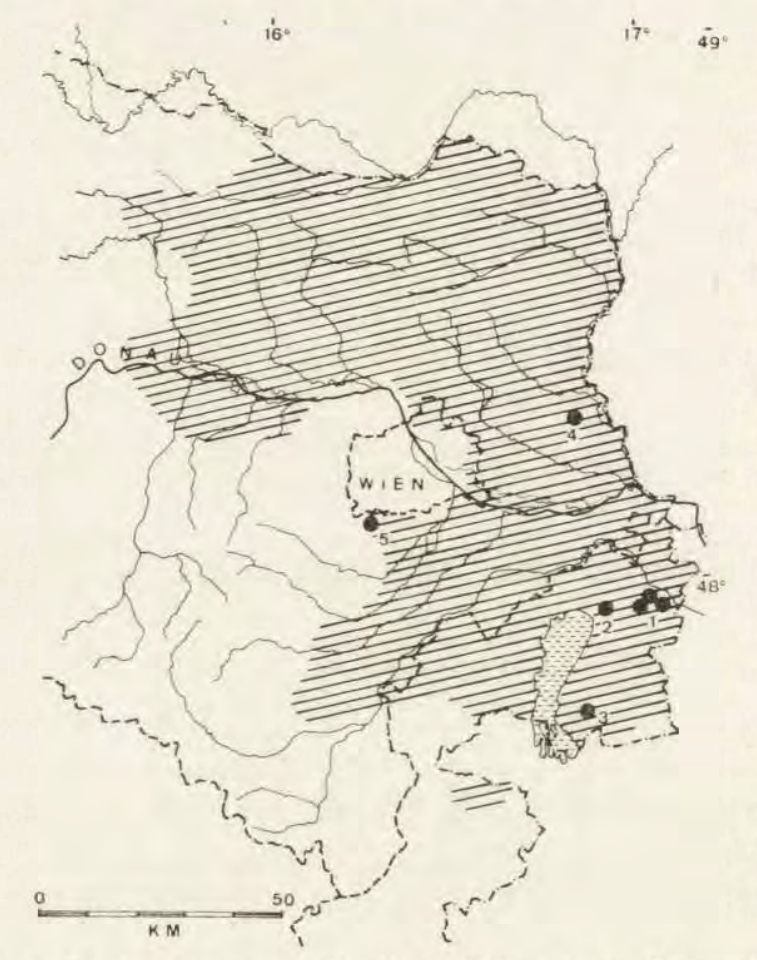

Abb. 1. Verbreitung von Citellus citellus L. in Österreich.

Beobachtet wurde hauptsächlich in folgenden Kolonien: 1. nahe dem Zurndorfer Eichenwald, Nickelsdorf. 2. „Panzergrabe” bei Neusiedl/See. 3. Apetloner Hutweide (Seewinkel), 4. Sandberg (Marchfeld), 5. Pechtoldsdorfer Heide.

den einerseits Weingärten, andererseits Reste ehemaliger Kolonien in erst kürzlich veränderten Biotopen (S t r a s c h il, 1972).

\section{MATERIAL UND METHODE}

Alle Untersuchungen fanden während der jährlichen Aktivitätsperiode von C. citellus zwischen Herbst 1968 und Herbst 1970 statt. Die Mehrzahl der Daten stamm aus den in Abb. 1 besonders markierten Gebieten.

Beobachtet wurde mit Hilfe eines Feldstechers Marke Swarovski Habicht $7 \times 42$ und einem Fernrohr Hartmann $30 \times 80$ Weitwinkel. Als Beobachtungsversteck diente teilweise ein Auto, vorwiegend fand aber ein zusammenlegbarer Hochstand mit einer Sitzhöhe von drei Metern Verwendung. 
Die Liste der Nahrungspflanzen resultiert aus 210 Direktbeobachtungen, bei denen die Pflanzen eindeutig identifiziert werden konnten. Die Analyse der tierischen Nahrungsbestandteile hingegen war nur in geringem Umfang durch Beobachtungen und Aufsammlungen von Fraßresten möglich. Dagegen konnten 170 Evertebraten aus 52 Aufsammlungen der jeweils frischen Losung im Bereich einer Kolonie sowie 7 Mageninhalten zumindest bis zur Familie oder Gattung, teilweise auch bis zur Art, bestimmt werden. Neun weitere Losungsproben enthielten nur unbestimmbare Chitinreste, und je eine weitere Losungs- und Magenprobe bestanden nur aus pflanzlichen Resten. Zu Vergleichszwecken wurden vor allem Insekten sowohl in Formolfallen als auch auf Probeflächen gesammelt.

\section{NAHRUNGSERWERB}

Die Hälfte bis zwei Drittel der Zeit, die die Tiere an der Erdoberfläche verbringen, nehmen Nahrungssuche und Nahrungsaufnahme in Anspruch. An den ersten und letzten Tagen ihrer Jahresaktivität entfernen sie sich dabei niemals weit von ihrem Bau. Für die übrige Zeit wurde ein Aktionsradius von durchschnittlich $60 \mathrm{~m}$ (19 Tagesbeobachtungen an Adulttieren) ermittelt; dieser kann aber in Einzelfällen beträchtlich erweitert werden. Ein Tier etwa trug mehrmals Getreidekörner von einem $150 \mathrm{~m}$ (Luftlinie) entfernten Getreidefeld in den Wohnbau ein.

Nach G r u li c h (1960) wird bei der Nahrungssuche offenbar vor allem der Geruchssinn eingesetzt. Besonders Pflanzen werden zuerst beschnüffelt, ehe sie stehengelassen oder mit den Zähnen abgepflückt werden. $\mathrm{Zu}$ hoch oder $\mathrm{zu}$ entfernt stehende Pflanzenteile werden dazu in der Männchenstellung mit den Vorderpfoten herangeholt. Auch sonst werden die Vorderpfoten vielfach beim Nahrungserwerb eingesetzt: eine oder beide Pfoten stopfen aus dem Maul hängende Pflanzenteile nach; sie halten größere Nahrungsbrocken (Getreideähren, Kirschen, Erdbeeren, Käfer, Raupen etc.) zum Abbeißen fest und drücken flüchtigere Beute, wie z.B. Heuschrecken, zu Boden, ehe sie auch mit dem Maul aufgenommen und beim Fressen wieder mit den Vorderpfoten festgehalten wird.

Getreidefelder stellen besondere Nahrungsplätze dar. Solange das Getreide noch niedrig ist (ca. bis zu $10-15 \mathrm{~cm}$ ) durchqueren die Tiere diese Felder, als wären sie eine Wiese und fressen an den verschiedensten Stellen. Wird das Getreide höher und die Halme kräftiger, so werden nur mehr die Ränder und schütteren Stellen aufgesucht. Um die Ähre $z u$ erreichen, wird ein Halm dann entweder mit den Vorderpfoten gebogen, bis sie in Männchenstellung mit den Zähnen am Ansatz abgepflückt werden kann, oder aber der Halm wird in $10-30 \mathrm{~cm}$ Höhe abgebissen und mit Hilfe der Vorderpfoten hinter den Incisivi durchgezogen, bis die Ähre beim Maul angelangt ist; manchmal wird danach noch der Stengelrest entfernt. Anschließend löst das Ziesel dann die einzelnen Körner heraus. 
Stacheln hindern die Tiere nicht daran, von einer Pflanze zu fressen. Junge Blätter von Ononis spinosa (Hauhechel) oder auch der Körbchenboden mancher Distelarten sind ein beliebtes Futter. In Ungarn holten sich die Tiere die noch geschlossenen Blütenknospen einer Carduus-Art aus einer Höhe bis zu $20 \mathrm{~cm}$, indem sie die obersten Blätter der Pflanze auseinander bogen und die Knospe von der Seite her abbissen.

Jede Nahrung wird entweder gleich an Ort und Stelle unter aufmerksamem Umherschauen verzehrt, oder aber zum nächstliegenden Wohnbau oder Fluchtloch getragen und davor sitzend gefressen. Bei nur geringen Störungen kommt es auch vor, daß ein Nahrungsbrocken in ein Loch mitgenommen und dort aufgefressen wird.

Der Transport der Nahrung erfolgt bei losen Sämereien und Körnern sowie klein zerbissenen Pflanzenteilen in den Backentaschen. Größere Stücke, wie Getreideähren, Käfer etc. werden im Maul halb herausstehend getragen.

$\mathrm{Ab}$ Mitte März sammelten die Tiere im Beobachtungsgebiet grüne Pflanzenteile und trugen sie in den Bau ein. Diese Sammeltätigkeit steigerte sich von Juli an und es schien dann auch das Schwergewicht mehr auf Samen und Körnern statt auf grünen Pflanzenteilen zu liegen. Eingetragene Nahrung wird im hiesigen Verbeitungsgebiet möglicherweise bald wieder verzehrt - jedenfalls wurden bei Grabungen an einigen Wohnbauen keine Vorräte gefunden.

Verschiedene Pflanzenteile machen zwar den Hauptteil der Zieselnahrung aus und kleine Insekten geraten manchmal wohl auch zufällig in den Magen - aber in vielen Fällen wird die tierische Nahrung auch richtig gesucht. So fraß ein Ziesel innerhalb von 30 Minuten sechs Raupen der Eule Tholera caespidis, die tagsüber in der Bodenoberfläche und unter Pflanzen verborgen liegen. Sie wurden jedesmal nach deutlichem Herumschnüffeln am Boden gefunden, während das Tier zwischendurch etwa $10 \mathrm{~cm}$ über dem Boden stehende Pflanzenteile verzehrte. Weiters wurden Ziesel beobachtet als sie oberflächliche Bauten von Lasius alienus (Formicidae) ausscharrten und auch kleinere Steine wegschoben, um an die darunterliegende Ameisenbrut heranzukommen. Elateridenlarven werden ebenfalls ausgegraben.

Ziesel beginnen bei Raupen an einem beliebigen Ende zu fressen bei Käfern ( $a b 5-10 \mathrm{~mm}$ Länge) beissen sie zuerst den Kopf und Thorax $\mathrm{ab}$ - das Abdomen wird weiterhin mit den Pfoten festgehalten und leergefressen.

\section{DIE NAHRUNG}

C. citellus frißt die verschiedensten Bestandteile von Pflanzen: Wurzeln, Stengel, Blätter, Blüten und Früchte. Auch die Auswahl der Nah- 
rungspflanzen umfaßt ein weites Spektrum (Tab. 1). Einige Pflanzengruppen (Gras- bzw. Getreide-, sowie Klee- und Luzernearten) werden von $C$. citellus, ähnlich wie vom verwandten $C$. suslicus $\mathrm{G}$ u el dens t a edt, 1780 (S u rdacki, 1960), deutlich bevorzugt. Besonders Getreidepflanzen oder Teile davon werden in praktisch allen Entwicklungsstadien gefressen:

(1) Pflänzchen, 5-10 cm groß: Blätter und oberste kleine Wurzeln, die an der Ansatzstelle noch aus der Erde ragen (das führt manchmal zur Verkümmerung der Pflanze).

(2) Stengel, schon kräftig, aber noch nicht ganz ausgewachsen: er wird in Einzelfällen in einer Höhe von $10-30 \mathrm{~cm}$ abgebissen und nach $\mathrm{Ab}$ schälen der äußeren Schichten das saftige Innere herausgefressen.

(3) Sowohl milchige als auch bereits trockene Getreidekörner. Für die deutliche Bevorzugung des Weizen vor Roggen (Tab. 1), mag auch die unterschiedliche Höhe, die die beiden Arten im Untersuchungsgebiet ausgewachsen erreichen, mitbestimmend sein. Dafür spricht zumindest, daß die Ähren der etwas verkümmerten und niedrigen Halme am Rande eines Roggenfeldes doch immer wieder gefressen werden.

Von anderen Feldfrüchten werden, nach Aussagen der Bauern, besonders Rüben durch Ziesel geschädigt: sie fressen die oberste Kappe der jungen Rüben ab.

Umfaßendere Schäden in der Landwirtschaft aber, wie sie aus anderen Ländern beschrieben werden ( $\mathrm{R}$ u ži ć-P etrov, 1950; T u r č ek 1963 u.a.), sind in Österreich nur aus der Zeit vor dem Zweiten Weltkrieg bekannt (Sc h w e ig er, 1955 u.a.).

Eine weitere Pflanze, die in diesem Rahmen hervorgehoben werden muß, ist der Löwenzahn (Leontodon sp.): $\mathrm{M} \circ \mathrm{hr}$ (1929) beschreibt Tiere, die in Gefangenschaft beim Anblick der gelben Löwenzahnblüten sehr erregt wurden und alle anderen Nahrungsangebote verschmähten. Diese Labordaten passen zu den Freilandbeobachtungen - die Löwenzahnblüten bildeten zeitweise wohl die Hauptnahrung, aber auch andere Teile der Pflanze sind sehr beliebt: (1) Blätter, im relativ geringsten Ausmaß. (2) Die erst wenig geöffneten Hüllblätter der Blütenstände. Sie werden aufgenommen indem das ganze Körbchen abgepflückt, mit den Vorderpfoten festgehalten und dann die äußersten grünen Hüllblätter mit den Zähnen heruntergeschält werden. (3) Ganze, voll erblühte oder halb verblühte Blütenstände. (4) Hüllblätter und Körbchenboden; oft zusammen mit einem Stück Stengel, mit unreifen aber ohne reife Samen.

In Tab. 1 sind die Futterpflanzen nach ihrer Beliebtheit geordnet und die Symbole derart gewählt, daß auch ersichtlich wird, ob der Pflanzenteil noch saftig oder bereits trocken war. Es wird deutlich, daß den 
Tabelle 1

Futterpflanzen von Citellus citellus L.

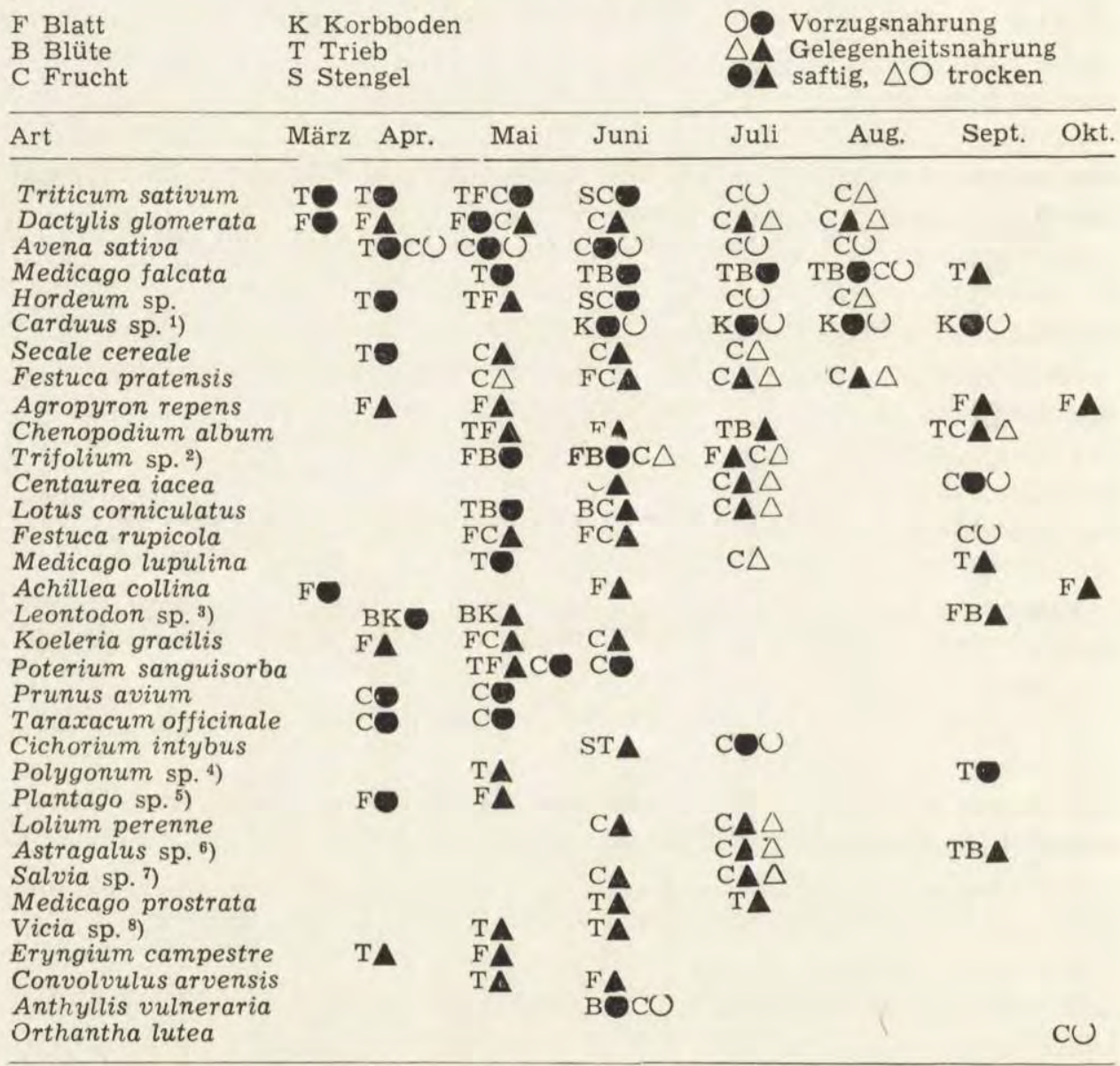

Vereinzelt wurden auch folgende Pflanzen gefressen:

Panicum sp. (T), Stipa pennata (C), Agrostis stolonifera (F, C), Poa pratensis angustifolia (C), Festuca pseudovina (C), Bromus mollis (C), Muscari racemosum (B), Stellaria media (T, B, C), Scleranthus polycarpos (T), Dianthus pontederae (C), Silene nutans (F), Raphanus raphanistrum (B), Potentilla arenaria (B), Fragaria viridis (C), Leguminosae (TF), Ononis spinosa (T, F), Coronilla varia (T), Phaseolus sp. (F), Acer campestris (T), Vitis vinifera (C), Pimpinella saxifraga (F), Teucrium chamaedrys (F), Galium verum (C), Bellis perennis (B), Hieracium pilosella (B). 1) Carduus nutans, C. acanthoides

2) Trifolin m campestre. T. repens, T. striatum, T. pratense

${ }^{3}$ Leontodon autumnalis, L. hispidum

4) Poly onum arenostrum, P. aviculare

5) Plantago major, $P$. lanceolata

6) Astragalus austriacus, A. onobrychis

7) Salvia austriaca, S. nemorosa, S. pratense

8) Vicia sp., V. lathyroides 
Tieren im hiesigen Verbreitungsgebiet praktisch während des ganzen Jahres ein gewisser Teil saftiger Nahrung zur Verfügung steht. Manchmal wird ja ein zu trockenes Futterangebot als Auslöser für einen Sommerschlaf genannt (K a l a bu k hov, 1956 nach T u r č ek, 1964); dieses Ergebnis paßt zu der Tatsache, daß ein solcher nicht festgestellt werden konnte.

Abschließend ist noch $\mathrm{zu}$ bemerken, daß gerade einige von Zieseln besonders bevorzugte Nahrungspflanzen (Plantago media, P. lanceolata, Leontodon hispidum, Taraxacum officinale) durch Beweidung besonders gefördert werden ( $\mathrm{T}$ is c hle r, 1965).

Tabelle 2

Häufigkeit einzelner Gruppen in den Bestimmungen der Chitinreste aus Magenund Losungsproben.

$\mathrm{n}=$ Anzahl der Identifizierungen. Daten aus Beobachtungen und Aufsammlungen im Anhang.

\begin{tabular}{lr}
\hline \multicolumn{1}{c}{ Tiergruppe } & $\%$ (n) \\
\hline $\begin{array}{l}\text { Coleoptera (Curculionidae, Carabidae, Scarabaeidae, M. melolontha, } \\
\text { Dytiscidae Larve, Coccinellidae, Chrysomelidae) }\end{array}$ & \\
Le pidoptera (Raupen, Nachtschmetterlinge, Lasiocampidae od. & \\
Arctiidae Raupe, Puppe) & $23(39)$ \\
Saltat oria (Larven. Acrididae, Tettigoniidae, Gryllidae, Liogryllus & \\
campestris) & $12(21)$ \\
Form ic idae (Tetramorium caespidum, Lasius alienus, Tapinoma & \\
erraticum, Solenopsis fugax, div. Puppen, Myrmica scabrinodis, & \\
M. rugulosa) & $11(18)$ \\
Insecta, unbestimmbar (Larven, Eier) & $6(10)$ \\
Araneae & $2(3)$ \\
Odonata (Kleinlibelle, Zygoptere) & $1(2)$ \\
Heteroptera, Hymenoptera & $1(2)$ \\
Diptera (Made) & $1(2)$ \\
\hline & $100(170)$ \\
\hline
\end{tabular}

Anhang: Tenebrionidae, Opatrum sabulosum, Otiorrhynchus sp., Cleonus sp., Phyllobius od. Polydrosus sp., Dorcadion fulvum, D. aethiops, D. pedestre, Amphimallus solistitialis, Elateridae Larve, Raupen (vorwiegend Tholera caespidis); Phasianus colchicus - vertrocknete Eier bzw. deren Inhalt, Saxicola torquata - ev. 1 ... aufgefunden.

In Tab. 2 sind die Ergebnisse der Bestimmung der Chitinreste zusammengefaßt. Insgesamt überwiegen Coleoptera in den Proben an Häufigkeit weit über Lepidoptera (Raupen), Saltatoria und Formicidae. Andere Gruppen nehmen nur minimale Prozentsätze ein. Bei einer Aufgliederung der Proben nach Jahreszeiten bleiben die Coleoptera an erster Stelle; im Frühjahr nehmen alle anderen Gruppen gemeinsam die zweite Stelie ein, im Sommer die Lepidoptera (Raupen) und im Herbst die Saltatoria. Diese Abfolge spiegelt weitgehend das jeweilige Angebot wieder. Be- 
obachtungen und Aufsammlungen zur tierischen Nahrung sind im Anhang zu Tab. 2 angeführt.

Vertebratenreste wurden nur in zwei Fällen festgestellt: ein Jungvogel von Saxicola torquata, von dem nicht bekannt ist, ob er vom Ziesel gefangen oder tot aufgefunden wurde; weiters fraßen mehrere Ziesel an vertrockneten Eihäutchen, Schalen und eventuell toten Kücken eines schon vor längerer Zeit zu Grunde gegangenen Geleges von Phasianus colchicus.

Frau Dr. F. Weiß-Spitzenberger und den Herren Prof. F. Schal1 er, Dipl. Ing. Dr. K. B a u er, Dr. W. F a ber, Dr. W. Hol zner, Prof, E. $\mathrm{H} \mathrm{übl}$, Dr. F. Kasy und Dr. H. Pruscha danke ich für die Unterstützung meiner Arbeit.

\section{LITERATUR}

1. B a u e r K., 1960: Die Säugetiere des Neusiedlerseegebietes. Bonner zool. Beitr. 11, 2-4: $141-344$.

2. Brinkmann M., 1951: Uber die Zieselkolonien in Oberschlesien. Bonner zool. Beitr. 2: 191-216.

3. Calinescu R. I., 1934: Taxonomische, biologische und biogeographische Forschungen über die Gattung Citellus Oken in Rumänien. Z. Säugetierkde., 9: 87-141.

4. Grulich I., 1960: Ground squirrel C. citellus L, in Czechoslovakia. Acta Acad. Scient. Cechosloven. Bas. Brun., 32: 473-563. [tschech. und engl. Zusf.].

5. Ka la bukhov N. I., 1956: Spjačka životnyh. [zit. nach Turček, 1964].

6. Mohr E., 1929: Meine beiden Ziesel (Citellus citellus L.). Zool. Garten, N. F. 1: $307-313$.

7. Pakizh V. I., 1958: Distribution of Citellus citellus L. in Moldavian SSR and certain data on its bionomics. Zool. Ž., 37: 105-119. [russisch].

8. Peshev Z., 1955: Investigations in systematics and biology of C. citellus $\mathrm{L}$. in Bulgaria. Bull. Inst. Zool. Acad. Scien. Bulg., 4-5: 277-327. [bulgar. und engl. Zusf.].

9. Ružić-Petrov A., 1950: Beitrag zur Kenntnis der Ökologie des Ziesels Citellus citellus L. Zbornik radova Instituta za Ekologiju i Biogeografiju, 1: 97-140. [serbokroat.].

10. Schweiger H., 1955: Tierische Schädlinge in Niederösterreich. Kartenblatt zum Atlas von Niederösterreich. Wien.

11. Straschil B., 1972: Citellus citellus L. (Europäisches Ziesel) in Ósterreich. (Zur Biologie und Okologie eines terrestrischen Säugetieres an der Grenze seines Verbreitungsgebietes). Dissertation am I. Zool. Inst. d. Univ. Wien.

12. Surdacki St., 1960: Untersuchungen über die Nahrung des Perlziesels, Citellus suslicus (G ülden ta e dt, 1780). Acta theriol., 4: 288-291.

13. T is chler W., 1965: Agrarökologie. Jena.

14. Turček F. J., 1955: On the distribution of the ground-squirrel Citellus citellus L. in Slovakia. Säugetierkundl. Mitt., 3, 1: 19-21.

15. Turček F. J., 1963: Beitrag zur Ókologie des Ziesels (Citellus citellus L.). II. Biologia, 18: 419-432. Bratislava.

16. Tur ček F. J., 1964: Food consumption in the European ground squirrel, with some remarks on the effects of Toxaphene. Ann. Zoology, IV, 8: 65-72. 
17. Werth E., 1934: Zur Verbreitung und Geschichte des Ziesels. Arb. Biol Reichsanst. f. Land- und Forstwirtsch., 21, 2: 255-267.

Eingegangen am 2 März, 1975.

Barbara HERZIG-STRASCHIL

POKARM I SPOSÓB JEGO ZDOBYWANIA U SUSŁA MORĘGOWANEGO

\section{Streszczenie}

Opisano upodobania pokarmowe susła moręgowanego Citellus citellus (L in n ae u s, 1766), przy czym listę zjadanych gatunków roślin ustalono tylko na podstawie obserwacji terenowych. Oznaczanie pokarmu zwierzęcego oparte było zarówno na obserwacjach w terenie i zbieraniu resztek pokarmowych, ale także pochodziło przez oznaczanie chitynowych resztek w kale i treści żołądka. Okazało się, że susły wykazują wyraźną preferencję niektórych rodzajów pokarmu, jakkolwiek lista gatunków roślin i zwierząt przez nie zjadanych jest długa. Podstawowym pokarmem są różne części roślin. Pokarm zwierzęcy składa się głównie z Coleoptera, następnie Lepidoptera (gąsienice) i Saltatoria. Przy pobieraniu pokarmu pomocną rolę spełniają kończyny przednie. 